\title{
Enrichment of neural-related genes in human mesenchymal stem cells from neuroblastoma patients
}

\author{
MIGUEL ÁNGEL RODRÍGUEZ-MILLA ${ }^{1}$, ISABEL MIRONES ${ }^{1}$, LUIS MARIÑAS-PARDO ${ }^{1}$, \\ GUSTAVO J. MELEN ${ }^{2}$, ISABEL CUBILLO ${ }^{1}$, MANUEL RAMÍREZ ${ }^{2}$ and JAVIER GARCÍA-CASTRO ${ }^{1}$ \\ ${ }^{1}$ Cellular Biotechnology Unit, Institute of Health Carlos III, Majadahonda, Madrid; \\ ${ }^{2}$ Department of Oncohematology, Hospital Niño Jesús, Madrid, Spain
}

Received January 25, 2012; Accepted March 22, 2012

DOI: $10.3892 / \mathrm{ijmm} .2012 .1008$

\begin{abstract}
Neuroblastoma (NB) is one of the most common pediatric solid tumors and, like most human cancers, is characterized by a broad variety of genomic alterations. Although mesenchymal stem cells (MSCs) are known to interact with cancer cells, the relationship between MSCs and metastatic NB cancercells in bone marrow (BM) is unknown. To obtain genetic evidence about this interaction, we isolated BM-derived MSCs from children with NB and compared their global expression patterns with MSCs obtained from normal pediatric donors, using the Agilent 44K microarrays. Significance analysis of microarray results with a false discovery rate (FDR) $<5 \%$ identified 496 differentially expressed genes showing either a 2-fold upregulation or downregulation between both groups of samples. Comparison of gene ontology categories of differentially expressed genes revealed the upregulation of genes categorized as 'neurological system process', 'cell adhesion', 'apoptosis', 'cell surface receptor linked signal transduction', 'intrinsic to membrane' and 'extracellular region'. Among the downregulated genes, several immunology-related terms were the most abundant. These findings provide preliminary genetic evidence of the interaction between MSCs and NB cancer cells in BM as well as identify relevant biological processes potentially altered in MSCs in response to NB.
\end{abstract}

\section{Introduction}

Neuroblastoma (NB), a poorly differentiated tumor derived from neural crest cells that affects mainly children, is the most common extracranial pediatric solid tumor. The origin of stroma in primary NB tumors, formed by Schwann cells, and whether the stroma is the cause or consequence of the

Correspondence to: Dr Javier García-Castro, Institute of Health Carlos III, lab. 51-00-031, Ctra Majadahonda-Pozuelo, km 2, E-28220 Majadahonda, Madrid, Spain

E-mail: jgcastro@isciii.es

Key words: neuroblastoma, metastasis, bone marrow, mesenchymal stem cells, tumor stroma, pediatric cancer maturation potential of tumor cells remain controversial. It has been hypothesized that crosstalk between Schwann cells and neuroblasts influences the biology and clinical behavior of NB tumors. However, little is known about the role of the NB microenvironment in metastasis localizations, especially in bone marrow (BM). Recent articles suggest that mesenchymal stem cells (MSCs) have a major role in maintaining stem cells niches in the BM (1), as well as in creating the tumor microenvironment (2). In this study we focused in BM-derived MSC from NB patients as a key factor in the development of metastasis.

During the past years several studies have used microarray-based high-throughput technologies to identify biological processes altered in NB cells. Hiyama et al (3) surveyed the differences in gene expression between unfavorable and maturing/regressing NB. Interestingly, in favorable $\mathrm{NB}$, neuronal differentiation signals such as $C D 44, I G F 2$, NTRKI and ANKI were overexpressed in maturing tumors. Similarly, Kamei et al (4) identified genes that exhibited altered gene expression in NB tumors associated with a favorable outcome. More recently, Chen et al (5) performed parallel global protein and mRNA expression profiling on NB tumors and identified that cell adhesion, nervous system development and cell differentiation processes were downregulated in stage $4 M Y C N$-amplified NB tumors, suggesting a less mature neural and a more invasive phenotype of this type of cells. Finally, Abel et al (6), based on expression profiling, identified 4 molecular subgroups of NB that can be distinguished by a 6-gene signature. These studies show that microarray techniques are useful tools for gene expression profiling in NB tumors.

It is well known that the BM is a common site for metastasis in patients with high-risk NB. Although little is known about the control of NB tumor growth by the BM microenvironment, there has been an increasing interest in the role of MSCs and their BM niches in cancer $(7,8)$. Some reports implicate MSCs having tumor-promoting effects whereas others show inhibition of tumor growth. Regarding the relationship between MSCs and NB, Ma et al (9) reported that MSCs in BM may enhance metastasis of NB via SDF-1/CXCR4 and SDF-1/ CXCR7 signaling. Moreover, CXCR5 may be involved in the attraction of human metastatic NB cells to the BM (10). Despite these findings, the relationship between MSCs and NB cancer cells is still unknown, and research in this area would add new scientific knowledge and provides new therapeutic ideas and 
targets. Thus, we isolated MSCs from BM of NB patients and control donors and compared their global expression patterns using microarrays. Our findings provide preliminary genetic evidence of the interaction between MSCs and NB cancer cells in $\mathrm{BM}$ as well as identify relevant biological processes potentially altered in MSCs in response to NB.

\section{Materials and methods}

Mesenchymal stem cell isolation, culture and characterization. MSCs were isolated from 4 NB pediatric patients (with no amplification of the N-Myc gene) and 4 healthy donors. The study protocol was approved by the Ethics Committee of the Hospital. All patients and volunteers were informed about the purpose of the study and provided written consent, by the parents or legal guardians, regarding their participation in the study. BM-derived MSCs were obtained by adherence to plastic. Mononucleated cells were obtained after centrifugation using a Ficoll-Paque gradient. Cells were cultured at $37^{\circ} \mathrm{C}$ with $5 \% \mathrm{CO}_{2}$ in DMEM (Lonza) supplemented with $10 \%$ fetal bovine serum (PAN-Biotech $\mathrm{GmbH})$. MSCs cultures were characterized according the International Society for Cellular Therapy criteria (11).

RNA isolation and $c R N A$ labeling. MSCs were stabilized in PrepProtect $^{\mathrm{TM}}$ (Miltenyi Biotec) and total-RNA was isolated using standard RNA extraction protocols (NucleoSpin ${ }^{\circledR}$ RNA II, Macherey-Nagel). RNA integrity and overall quality was checked via the Agilent 2100 Bioanalyzer expert software (Agilent Technologies). All RNA samples revealed an RNA Integrity Number (RIN) between 7.3 and 10. For the linear T7-based amplification step, $1 \mu \mathrm{g}$ of each total-RNA sample was used. To produce Cy3-labeled cRNA, the RNA samples were amplified and labeled using the Agilent Low RNA Input Linear Amp kit (Agilent Technologies) following the manufacturer's protocol. Yields of cRNA and the dye-incorporation rate were measured with the ND-1000 Spectrophotometer (NanoDrop Technologies).

Microarray hybridization. The hybridization procedure was performed according to the Agilent 60-mer oligo microarray processing protocol using the Agilent Gene Expression Hybridization kit (Agilent Technologies). Briefly, $1.65 \mu \mathrm{g}$ Cy3-labeled fragmented cRNA in hybridization buffer was hybridized overnight $\left(17 \mathrm{~h}, 65^{\circ} \mathrm{C}\right)$ to Agilent Whole Human Genome Oligo Microarrays 4x44K using Agilent's recommended hybridization chamber and oven. Finally, the microarrays were washed once with $6 \mathrm{X}$ SSPE buffer containing $0.005 \% \mathrm{~N}$-lauroylsarcosine for $1 \mathrm{~min}$ at room temperature followed by a second wash with preheated 0.06X SSPE buffer $\left(37^{\circ} \mathrm{C}\right)$ containing $0.005 \% \mathrm{~N}$-lauroylsarcosine for $1 \mathrm{~min}$. The last washing step was performed with acetonitrile for $30 \mathrm{sec}$. Fluorescence signals of the hybridized Agilent microarrays were detected using Agilent's Microarray Scanner System (Agilent Technologies). The Agilent Feature Extraction Software (FES) was used to read out and process the microarray image files.

Microarray data analysis. The microarray raw data have been deposited at the NCBI Gene Expression Omnibus under the accession number GSE35133 (http://www.ncbi.nlm.nih.gov/

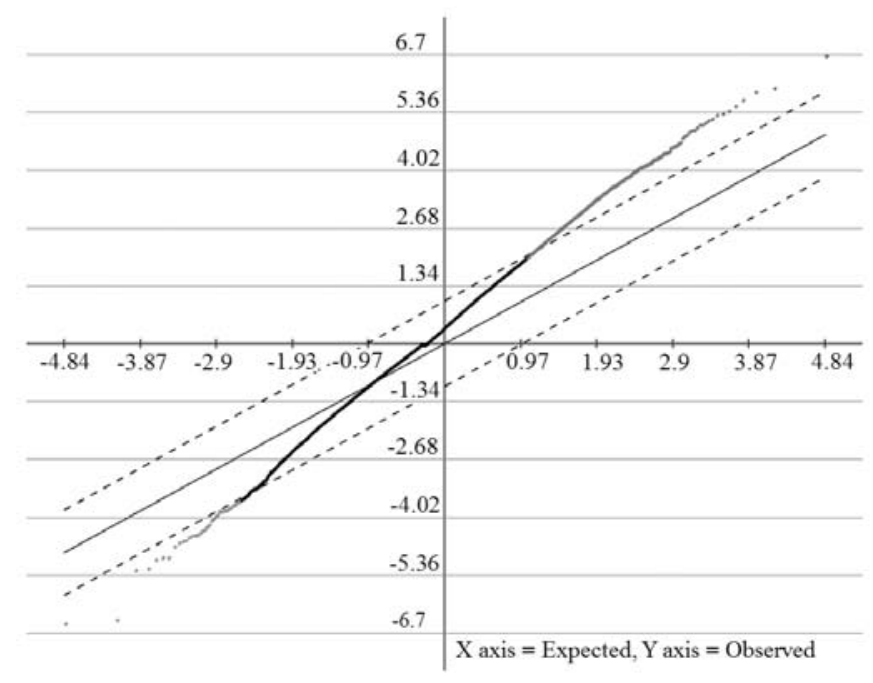

Figure 1. Significance analysis of microarrays (SAM) plot from the comparison of NB wih control. The plot identifies genes which are significantly different across the groups (2-fold upregulation or downregulation and FDR $<0.05 \%$ ). Spots above the upper dotted line indicate significantly upregulated genes and spots below the lower dotted line indicate significantly downregulated genes.

geo/query/acc.cgi?token=rdszfaigqkmgqdw\&acc=GSE35133). Intensity values of flagged spots below background were filtered out, data were normalized using the array median and the mean values of 3 replicates for each biological sample were calculated. Statistical analysis of microarray significance (SAM) was performed to identify genes with significant changes in expression, and permutations were used to estimate the false discovery rate (FDR) (12). Genes were selected if they showed a 2 -fold regulation and a FDR $<0.05 \%$. These statistical analyses were performed using TMEV and default parameters were used unless specified (13).

Gene ontology $(G O)$ analysis. To better characterize the functionally-related genes which showed at least a 2-fold regulation in the microarray analysis, the genes were assigned to Gene Ontologies using a database for annotation, visualization and integrated discovery (DAVID) (14). We used the 3 ontologies produced by the GO consortium, namely 'biological process', 'cellular component' and 'molecular function'. GO terms were collected, redundant terms were excluded, and P-values were used to evaluate the significance of the terms.

\section{Results}

We isolated MSCs from NB patients (NB-MSCs) as well as from normal donors. All MSC cultures were characterized according to the International Society for Cellular Therapy criteria: morphology, positive/negative markers and differentiation properties (data not shown) (11). We analyzed the expression profiles of RNA from NB-MSCs compared to those from normal donors, using the Agilent Whole Human Genome Oligo Microarrays. The results from the SAM analysis ( $\geq 2$-fold regulation and FDR $<0.05 \%$ ) revealed that 454 genes had transcript levels significantly higher in NB-MSCs, whereas 42 genes had transcript levels significantly lower in NB-MSCs (Fig. 1). A list of the genes showing the strongest upregulation 
Table I. List of upregulated genes in hMSCs from NB patients.

\begin{tabular}{|c|c|c|c|c|}
\hline Gene symbol & Gene description & $\begin{array}{c}\text { GenBank } \\
\text { accession no. }\end{array}$ & $\begin{array}{l}\text { Fold- } \\
\text { change }\end{array}$ & Q-value \\
\hline GPR128 & G protein-coupled receptor 128 & NM_032787 & 44.269 & 4.5173 \\
\hline TMEFF2 & $\begin{array}{l}\text { Transmembrane protein with EGF-like and } \\
\text { two follistatin-like domains } 2\end{array}$ & NM_016192 & 18.655 & 0.7810 \\
\hline ANXA10 & Annexin A10 & NM_007193 & 13.700 & 1.2968 \\
\hline ITGA2 & Integrin, $\alpha 2$ (CD49B, $\alpha 2$ subunit of VLA-2 receptor) & NM_002203 & 11.435 & 0.0000 \\
\hline A_32_P208076 & Unknown & A_32_P208076 & 10.707 & 0.0000 \\
\hline A_24_P365349 & Unknown & A_24_P365349 & 9.664 & 1.6333 \\
\hline THC2402993 & Unknown & THC2402993 & 9.011 & 0.0000 \\
\hline NEF3 & Neurofilament 3 (150 kDa medium) & NM_005382 & 8.468 & 1.0034 \\
\hline IGFBP1 & Insulin-like growth factor binding protein 1 , transcript variant 1 & NM_000596 & 8.255 & 4.6632 \\
\hline WDR69 & WD repeat domain 69 & NM_178821 & 8.132 & 3.9950 \\
\hline C1orf110 & Chromosome 1 open reading frame 110 & ВC040018 & 7.958 & 3.6213 \\
\hline TREM1 & Triggering receptor expressed on myeloid cells 1 & NM_018643 & 7.931 & 4.5173 \\
\hline FLJ37228 & cDNA FLJ37228 fis, clone BRAMY2000411 & AK094547 & 7.177 & 0.0000 \\
\hline KCTD4 & Potassium channel tetramerisation domain containing 4 & NM_198404 & 7.162 & 4.2453 \\
\hline CNIH3 & Cornichon homolog 3 & NM_152495 & 6.984 & 3.9107 \\
\hline ENST00000379108 & Unknown & ENST00000379108 & 6.882 & 3.3557 \\
\hline CALB2 & Calbindin 2, $29 \mathrm{kDa}$ (calretinin) & NM_001740 & 6.503 & 4.3242 \\
\hline COL4A5 & Collagen, type IV, $\alpha 5$, transcript variant 2 & NM_033380 & 6.438 & 2.1503 \\
\hline SLC6A15 & Solute carrier family 6 , member 15 , transcript variant 1 & NM_182767 & 6.234 & 0.9502 \\
\hline TSPAN8 & Tetraspanin 8 & NM_004616 & 5.955 & 1.4171 \\
\hline SLC7A14 & mRNA for KIAA1613 protein & AB046833 & 5.889 & 0.0000 \\
\hline THC2438492 & Unknown & THC2438492 & 5.588 & 0.0000 \\
\hline ENST00000222543 & Similar to tissue factor pathway inhibitor 2 precursor (TFPI-2) & ENST00000222543 & 5.577 & 3.2311 \\
\hline PSG7 & Pregnancy specific $\beta$-1-glycoprotein 7 & NM_002783 & 5.553 & 2.6056 \\
\hline DCBLD2 & Discoidin, CUB and LCCL domain containing 2 & NM_080927 & 5.547 & 0.7810 \\
\hline HTR1F & 5-hydroxytryptamine (serotonin) receptor 1F & NM_000866 & 5.539 & 1.6929 \\
\hline SLC7A14 & Solute carrier family 7, member 14 & NM_020949 & 5.424 & 1.2573 \\
\hline RNF128 & Ring finger protein 128, transcript variant 1 & NM_194463 & 5.387 & 4.2453 \\
\hline SHC3 & SHC (Src homology 2 domain containing) transforming protein 3 & NM_016848 & 5.329 & 2.0866 \\
\hline HLA-DR B & HLA class II DR- $\beta$ & X12544 & 5.313 & 3.5143 \\
\hline SAMD3 & Sterile $\alpha$ motif domain containing 3 , transcript variant 2 & NM_152552 & 5.254 & 0.0000 \\
\hline SEMA3E & Semaphorin 3E & NM_012431 & 5.227 & 2.0866 \\
\hline LOC284344 & Similar to biliary glycoprotein 1 precursor & AK097672 & 5.197 & 4.8517 \\
\hline PSG4 & Pregnancy specific $\beta$-1-glycoprotein 4 , transcript variant 2 & NM_213633 & 5.164 & 1.2496 \\
\hline RGS4 & Regulator of G-protein signaling 4 & NM_005613 & 5.151 & 1.4010 \\
\hline AREG & Amphiregulin (schwannoma-derived growth factor) & NM_001657 & 5.043 & 4.0968 \\
\hline DNER & Delta-notch-like EGF repeat-containing transmembrane & NM_139072 & 4.943 & 2.4280 \\
\hline AK094786 & cDNA FLJ37467 fis, clone BRAWH2011920 & AK094786 & 4.787 & 1.2384 \\
\hline HGD & Homogentisate 1,2-dioxygenase (homogentisate oxidase) & NM_000187 & 4.784 & 1.8692 \\
\hline RGS18 & Regulator of G-protein signaling 18 & NM_130782 & 4.752 & 4.6632 \\
\hline SAMD3 & Sterile $\alpha$ motif domain containing 3 , transcript variant 1 & NM_001017373 & 4.749 & 1.1850 \\
\hline SLC24A3 & Solute carrier family 24, member 3 & NM_020689 & 4.736 & 3.4520 \\
\hline F2RL1 & Coagulation factor II (thrombin) receptor-like 1 & NM_005242 & 4.683 & 2.6953 \\
\hline CST1 & Cystatin SN & NM_001898 & 4.625 & 1.6333 \\
\hline TMEM158 & Transmembrane protein 158 & NM_015444 & 4.618 & 3.4313 \\
\hline THC2455389 & ORF2280 gene homolog & THC2455389 & 4.608 & 3.1260 \\
\hline AK127194 & cDNA FLJ45259 fis, clone BRHIP2020695 & AK127194 & 4.588 & 3.1367 \\
\hline PSCDBP & Pleckstrin, Sec7 and coiled-coil domains, binding protein & NM_004288 & 4.539 & 2.4280 \\
\hline CLGN & Calmegin & NM_004362 & 4.533 & 3.7978 \\
\hline RP11-138L21.1 & Similar to contactin associated protein (Caspr) & AK054645 & 4.436 & 2.3612 \\
\hline BCAN & Brevican & ВC005081 & 4.370 & 0.0000 \\
\hline NPTX1 & Neuronal pentraxin I & NM_002522 & 4.290 & 2.8954 \\
\hline HLA-DRB5 & Major histocompatibility complex, class II, DR $\beta 5$ & NM_002125 & 4.284 & 3.3557 \\
\hline SULT4A1 & Sulfotransferase family $4 \mathrm{~A}$, member 1 & NM_014351 & 4.246 & 2.5183 \\
\hline THC2335868 & ALU5_HUMAN (P39192) Alu subfamily SC & THC2335868 & 4.231 & 0.0000 \\
\hline FATE1 & Fetal and adult testis expressed 1 & NM_033085 & 4.196 & 4.3477 \\
\hline
\end{tabular}


Table II. List of downregulated genes in hMSCs from NB patients.

\begin{tabular}{|c|c|c|c|c|}
\hline Gene symbol & Gene description & $\begin{array}{c}\text { GenBank } \\
\text { accession no. }\end{array}$ & $\begin{array}{l}\text { Fold- } \\
\text { change }\end{array}$ & Q-value \\
\hline IFI27 & Interferon, $\alpha$-inducible protein 27 & NM_005532 & 0.0487 & 4.052 \\
\hline CRIP1 & Cysteine-rich protein 1 (intestinal) & NM_001311 & 0.0994 & 4.324 \\
\hline CCL8 & Chemokine (C-C motif) ligand 8 & NM_005623 & 0.1021 & 3.438 \\
\hline LSP1 & Lymphocyte-specific protein 1 , transcript variant 3 & NM_001013254 & 0.1188 & 1.462 \\
\hline CDCA7 & Cell division cycle associated 7 , transcript variant 1 & NM_031942 & 0.1509 & 0.000 \\
\hline ENST00000372045 & cDNA clone CS0DI016YJ18 (CR623913) & ENST00000372045 & 0.1757 & 2.385 \\
\hline IL21R & Interleukin 21 receptor, transcript variant 2 & NM_181078 & 0.1975 & 4.052 \\
\hline $\mathrm{C} 2$ & Complement component 2 & NM_000063 & 0.2005 & 4.448 \\
\hline MT1JP & MTB & AF348994 & 0.2014 & 4.517 \\
\hline IFITM1 & Interferon induced transmembrane protein 1 (9-27) & NM_003641 & 0.2049 & 3.995 \\
\hline ENST00000313624 & cDNA clone DKFZp667P0410 (AL831953) & ENST00000313624 & 0.2118 & 3.438 \\
\hline FAM70A & Family with sequence similarity 70 , member A & NM_017938 & 0.2231 & 2.385 \\
\hline ISG20 & Interferon stimulated exonuclease gene $20 \mathrm{kDa}$ & NM_002201 & 0.2419 & 2.385 \\
\hline DKFZP761M1511 & cDNA FLJ39342 fis, clone OCBBF2018873 & AK096661 & 0.2459 & 3.995 \\
\hline MBOAT1 & cDNA FLJ16207 fis, clone CTONG2019822 & AK131269 & 0.2475 & 0.000 \\
\hline $\mathrm{JPH} 2$ & Junctophilin 2 , transcript variant 1 & NM_020433 & 0.2524 & 1.448 \\
\hline $\mathrm{C} 1 \mathrm{R}$ & Complement component $1, \mathrm{r}$ subcomponent & NM_001733 & 0.2639 & 4.724 \\
\hline ENPP2 & $\begin{array}{l}\text { Ectonucleotide pyrophosphatase/phosphodiesterase } 2 \\
\text { (autotaxin), transcript variant } 1\end{array}$ & NM_006209 & 0.2789 & 1.462 \\
\hline TMEM119 & Transmembrane protein 119 & NM_181724 & 0.2826 & 2.385 \\
\hline E2F2 & E2F transcription factor 2 & NM_004091 & 0.2863 & 2.385 \\
\hline OLFML2B & Olfactomedin-like 2B & NM_015441 & 0.2879 & 2.854 \\
\hline EXO1 & Exonuclease 1 , transcript variant 3 & NM_003686 & 0.2881 & 2.487 \\
\hline CCNE2 & Cyclin E2, transcript variant 1 & NM_057749 & 0.2951 & 1.448 \\
\hline C12orf46 & Chromosome 12 open reading frame 46 & NM_152321 & 0.3028 & 2.385 \\
\hline ECGF1 & Endothelial cell growth factor 1 (platelet-derived) & NM_001953 & 0.3067 & 1.462 \\
\hline SLC2A12 & Solute carrier family 2 (facilitated glucose transporter), member 12 & NM_145176 & 0.3321 & 4.724 \\
\hline NAV2 & Steerin3 protein, alternative exon $1 \mathrm{~b}$ & AJ488202 & 0.3387 & 3.145 \\
\hline A_24_P927205 & Unknown & A_24_P927205 & 0.3480 & 4.052 \\
\hline IL7 & Interleukin 7 & NM_000880 & 0.3811 & 4.463 \\
\hline ENST00000270031 & Unknown & ENST00000270031 & 0.3965 & 4.448 \\
\hline GBP1 & Guanylate binding protein 1, interferon-inducible, $67 \mathrm{kDa}$ & NM_002053 & 0.4015 & 2.385 \\
\hline RAB42 & RAS oncogene family & NM_152304 & 0.4214 & 4.517 \\
\hline HELLS & Helicase, lymphoid-specific & NM_018063 & 0.4240 & 4.448 \\
\hline BARD1 & BRCA1 associated RING domain 1 & NM_000465 & 0.4244 & 3.699 \\
\hline RAD51AP1 & RAD51 associated protein 1 & NM_006479 & 0.4256 & 2.392 \\
\hline FLJ39660 & cDNA clone DKFZp434P055 & AL834537 & 0.4292 & 2.576 \\
\hline HIRA & Histone cell cycle regulation defective homolog $\mathrm{A}$ & NM_003325 & 0.4327 & 4.448 \\
\hline THC2376015 & Unknown & THC2376015 & 0.4385 & 4.168 \\
\hline POLE2 & Polymerase (DNA directed), $\varepsilon 2$ (p59 subunit) & NM_002692 & 0.4623 & 2.854 \\
\hline GBP2 & Guanylate binding protein 2 , interferon-inducible & NM_004120 & 0.4708 & 4.183 \\
\hline ATAD2 & ATPase family, AAA domain containing 2 & NM_014109 & 0.4838 & 4.069 \\
\hline
\end{tabular}

in NB-MSCs is shown in Table I whereas the downregulated genes are shown in Table II.

To illustrate the differences between NB and normal samples the genes whose expression was induced or repressed by at least 2.5 -fold in NB samples are shown as heat map in the Fig. 2, where NB and normal samples are clearly differentiated. Interestingly, several of the genes are known to play roles in NB (ANXA10, ITGA2, COL4A5 and SHC3) or other types of cancer (TMEFF2, TSPAN8, DCBLD2, PSCDBP and
BCAN) (Table I). Table $\mathrm{V}$ lists the genes involved in neuronal processes that were $>2.0$-fold upregulated.

To further examine the differences in the expression profiles between NB-MSCs and normal donors, the 496 significantly up or downregulated genes were analyzed with the DAVID software and classified into the 3 main GO domains. For the upregulated genes, in the gene ontology 'biological process' we identified 138 terms, in 'cellular component' 23 terms, and in 'molecular function' 17 terms. The highest ranked terms 


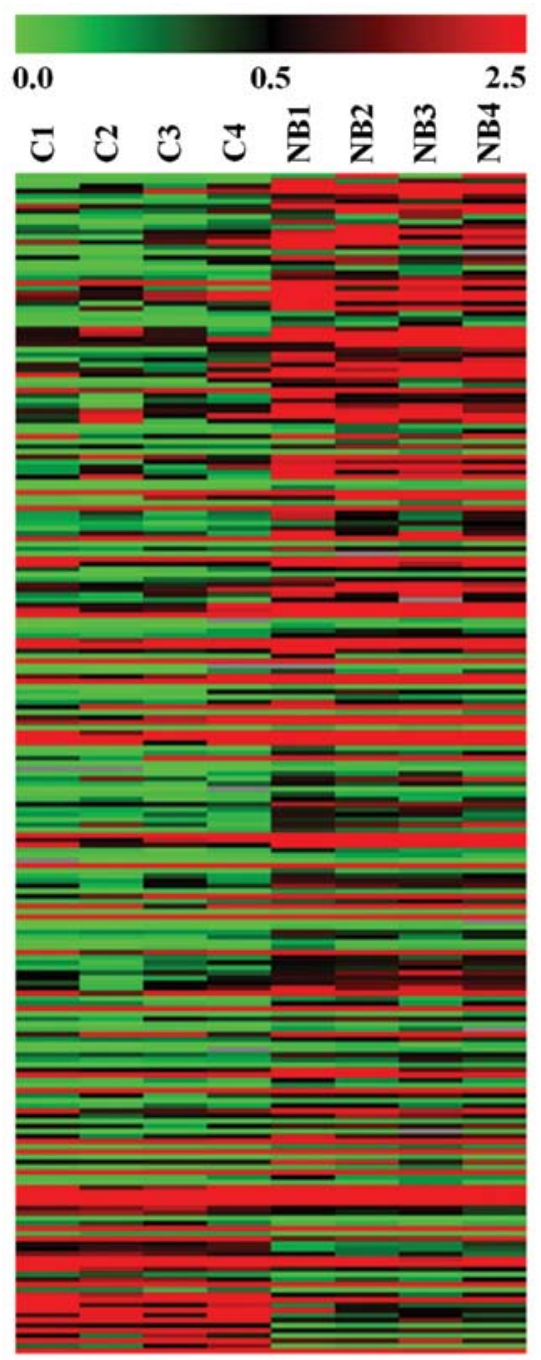

Figure 2. Heat map analysis of microarray data by SAM. Only genes undergoing a 2.5-fold (up or down) differential expression between control (C) and neuroblastoma (NB) samples (FDR $<0.05 \%$ ) are shown.

are shown in Table III. For the downregulated genes, in the gene ontology 'biological process' we identified 24 terms, in 'cellular component' 1 term, and in 'molecular function' 9 terms. The highest ranked terms are shown in Table IV.

\section{Discussion}

The role of MSCs in tumor progression is unclear as it has been suggested that MSCs may promote or suppress tumor growth (8). Therefore, identifying potential genes regulated in BM-derived MSCs by NB cancer cells would be of great importance to assess their role in determining disease outcome. Previously, Hahn et al (15) studied the effect of conditioned medium of BM cultures in NB cell growth in vitro (15). They showed that BM cultures may stimulate the proliferation and differentiation suppression of NB cells. In this model, monocytes seem to be the mediators of these effects. However, there is no data on how NB cells modify the characteristics of BM-resident cell populations. In this study, we analyzed for the first time the expression profiles of BM-derived MSCs from NB patients and report the identification of 496 genes with more than a 2-fold increase or decrease transcript levels.
Our findings suggest that NB cancer cells may have an impact on several processes of MSCs localized in BM.

Interestingly, our microarray analysis revealed that some of the top ranked upregulated genes in NB-MSCs (ANXA10, ITGA2, COL4A5 and SHC3) have been previously reported to have a potential role in NB (Table I). For instance, Annexin A10 (ANXA10) has been identified in a microarray analysis of human NB stem cells as a gene associated with malignancy (16). Similarly, integrin upregulation has been reported as a marker of NB cell differentiation (17). The same study identified the overexpression of COL4A5 in unfavorable NB. Finally, a distinct role of ShcC (SHC3) docking protein in the differentiation of NB has been proposed (18). In addition, we identified several genes reported in other studies to be regulated in different types of cancer: TMEFF2 $(19,20)$, TSPAN8 $(21,22)$, DCBLD2 $(23,24)$. Taken together, these observations suggest that the interaction between MSCs and NB cancer cells in the BM microenvironment induces changes in the expression of cancer-related genes in the MSCs. An unlikely explanation would be the cellular fusion of NB cells and MSCs, in a similar manner to Rizvanov et al (25) who observed rare in vitro cell fusion in co-cultures of NB tumor cells and MSCs.

The GO functional classification analysis through DAVID showed a number of mainly affected categories further suggesting that NB-MSCs are altered (Table III). Overall, we noted that in the 'cellular component' domain, NB upregulated a large number of genes encoding proteins 'intrinsic to membrane' $(n=98)$ and localized to the 'extracellular region' $(n=54)$, suggesting that NB cancer cells may exert a large repertoire of changes in these MSCs compartments. It is likely that functional relationships between NB and MSCs are mostly mediated through these proteins. Analysis of the category 'biological process' revealed effects on MSCs in terms previously described in NB cancer cells in the literature. Most importantly, we remark on the upregulation of genes in the term 'neurological system processes'. Despite of the wide variety of proteins encoded by these genes, they may provide insights into potential neurological functions altered in NB-MSCs. Interestingly, regulation of neural-related genes has also been shown in previous microarray analysis of NB tumors. Thus, Hiyama et al (3) reported that in favorable NB neuronal differentiation signals were overexpressed in maturing tumors whereas Chen et al (5) found that in NB tumors of stage $4^{+}$, proteins with functions in nervous system development were downregulated, suggesting a less mature neural and a more invasive phenotype of these tumors.

The absence of NB markers in our MSC cultures pants to the absence of a tumor cell contamination. Then, it is tempting to speculate whether our findings reflect that MSCs in the $\mathrm{BM}$ microenvironment of $\mathrm{NB}$ patients redirect toward neuronal lineage. Therefore, MSCs have been proposed to adopt neural cell phenotypes, although this occurs at a very low frequency (26). In this sense, it has been proposed that NB cells would induce MSCs differentiation into Schwann cells (27). However in our data we did not observe an increase of classical Schwann-markers such as S100, Egr-1 or Egr-2 in NB-MSCs. On the other hand, MSCs would suffer a dedifferentiation process since a neuroectodernal origin of fetal MSCs localised in BM has been proposed (28). 

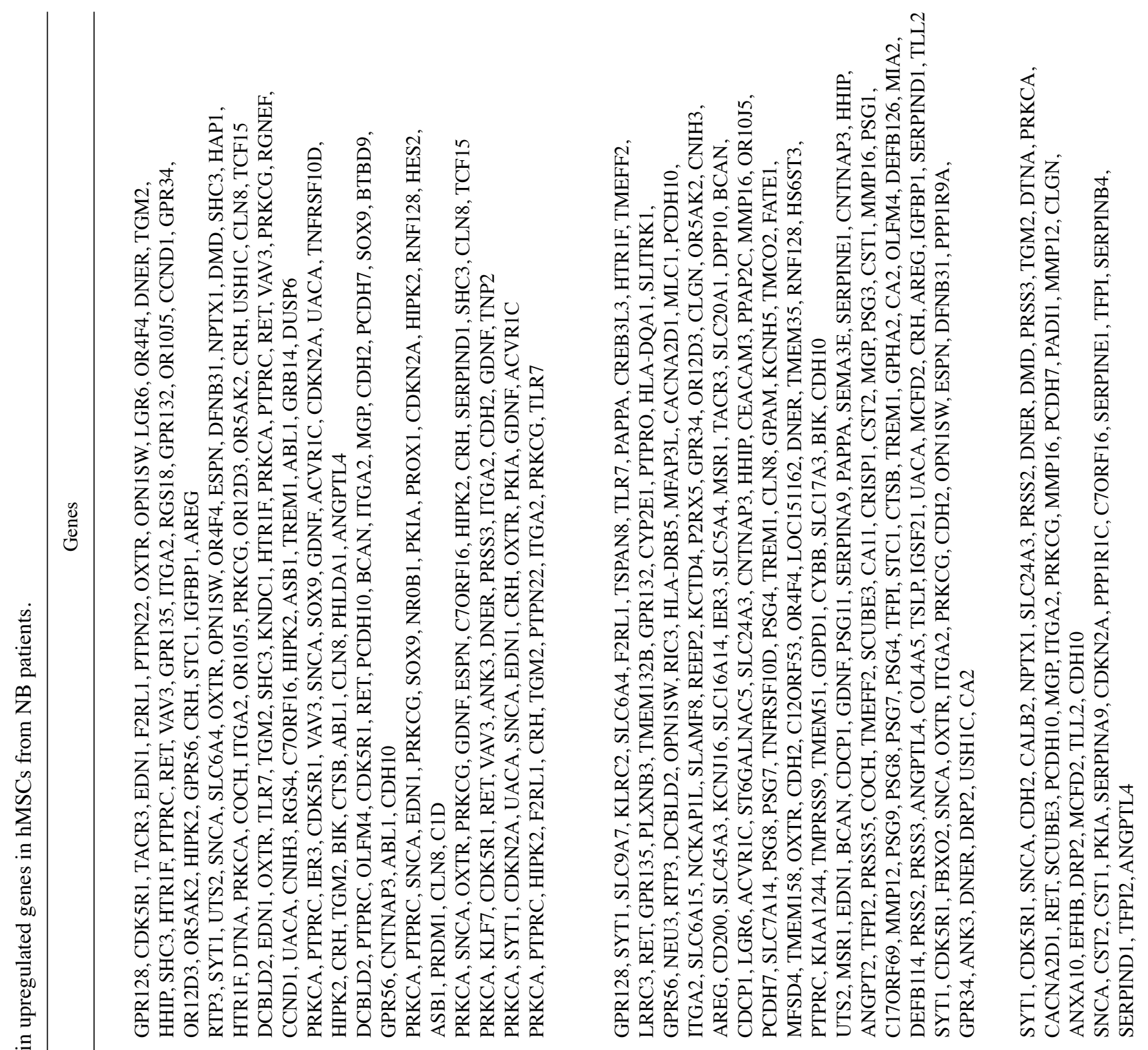
Table IV. Main GO terms of the different GO categories enriched in downregulated genes in hMSCs from NB patients.

\begin{tabular}{llll}
\hline GO term & Count & P-value & \multicolumn{1}{c}{ Genes } \\
\hline $\begin{array}{l}\text { Biological process } \\
\text { GO:0006955. Immune response }\end{array}$ & 9 & 0.00003 & EXO1, IL7, ENPP2, CCL8, RSAD2, C1R, C2, GBP2, GBP1 \\
GO:0006259. DNA metabolic process & 7 & 0.003 & EXO1, CCNE2, RAD51AP1, POLE2, HELLS, ISG20, BARD1 \\
GO:0006952. Defense response & 5 & 0.02 & LSP1, CCL8, RSAD2, C1R, C2 \\
GO:0007049. Cell cycle & 5 & 0.06 & EXO1, CCNE2, E2F2, HELLS, BARD1 \\
GO:0006959. Humoral immune response & 4 & 0.0004 & EXO1, IL7, C1R, C2 \\
GO:0002252. Immune effector process & 4 & 0.002 & EXO1, RSAD2, C1R, C2 \\
GO:0046649. Lymphocyte activation & 4 & 0.01 & EXO1, IL7, IL21R, HELLS \\
GO:0006281. DNA repair & 4 & 0.01 & EXO1, RAD51 AP1, POLE2, BARD1 \\
\hline
\end{tabular}

Table V. List of neuronal-related genes upregulated in hMSCs from NB patients.

\begin{tabular}{|c|c|c|c|c|}
\hline $\begin{array}{l}\text { Gene } \\
\text { symbol }\end{array}$ & Gene description & $\begin{array}{c}\text { GenBank } \\
\text { accession no. }\end{array}$ & $\begin{array}{c}\text { Fold } \\
\text { change }\end{array}$ & Q-value \\
\hline ITGA2 & Integrin, $\alpha 2$ (CD49B, $\alpha 2$ subunit of VLA-2 receptor) & NM_002203 & 11.435 & 0.0000 \\
\hline HTR1F & 5-hydroxytryptamine (serotonin) receptor $1 \mathrm{~F}$ & NM_000866 & 5.539 & 1.6929 \\
\hline SHC3 & SHC (Src homology 2 domain containing) transforming protein 3 & NM_016848 & 5.329 & 2.0866 \\
\hline NPTX1 & Neuronal pentraxin I & NM_002522 & 4.290 & 2.8954 \\
\hline DFNB31 & Autosomal recessive deafness type 31 protein 2 & AK056190 & 3.487 & 0.0000 \\
\hline SYT1 & Synaptotagmin I & NM_005639 & 3.452 & 4.6886 \\
\hline RTP3 & Receptor transporter protein 3 & NM_031440 & 3.282 & 2.3923 \\
\hline OXTR & Oxytocin receptor & NM_000916 & 3.274 & 3.5862 \\
\hline SNCA & Synuclein, $\alpha$ (non A4 component of amyloid precursor) & NM_007308 & 2.980 & 2.8954 \\
\hline ESPN & Espin & NM_031475 & 2.518 & 1.5910 \\
\hline $\mathrm{CRH}$ & Corticotropin releasing hormone & NM_000756 & 2.496 & 3.3557 \\
\hline CLN8 & Ceroid-lipofuscinosis, neuronal 8 (epilepsy, progressive with mental retardation) & NM_018941 & 2.479 & 1.0034 \\
\hline $\mathrm{COCH}$ & Coagulation factor $\mathrm{C}$ homolog, cochlin & NM_004086 & 2.420 & 3.6986 \\
\hline DMD & Dystrophin (muscular dystrophy, Duchenne and Becker types) & NM_004010 & 2.408 & 4.0521 \\
\hline DTNA & Dystrobrevin, $\alpha$, transcript variant 1 & NM_001390 & 2.330 & 2.4868 \\
\hline HAP1 & Huntingtin-associated protein 1 (neuroan 1), transcript variant 1 & NM_003949 & 2.311 & 2.1529 \\
\hline OR12D3 & Olfactory receptor, family 12 , subfamily $\mathrm{D}$, member 3 & NM_030959 & 2.301 & 1.0207 \\
\hline OR5AK2 & Olfactory receptor, family 5 , subfamily AK, member 2 & NM_001005323 & 2.293 & 0.7810 \\
\hline TCF15 & Transcription factor 15 (basic helix-loop-helix) & NM_004609 & 2.151 & 2.5183 \\
\hline PRKCG & Protein kinase $\mathrm{C}, \gamma$ & NM_002739 & 2.138 & 3.4520 \\
\hline PRKCA & Protein kinase $\mathrm{C}, \alpha$ & NM_002737 & 2.132 & 1.3963 \\
\hline OR4F4 & Olfactory receptor, family 4 , subfamily $\mathrm{F}$, member 4 & NM_001004195 & 2.104 & 1.6012 \\
\hline OPN1SW & Opsin 1 (cone pigments), short-wave-sensitive (color blindness, tritan) & NM_001708 & 2.069 & 1.1850 \\
\hline UTS2 & Urotensin 2 , transcript variant 1 & NM_021995 & 2.067 & 1.3372 \\
\hline OR10J5 & Olfactory receptor, family 10 , subfamily $\mathrm{J}$, member 5 & NM_001004469 & 2.052 & 1.0034 \\
\hline USH1C & cDNA: FLJ21290 fis, clone COL01954 & AK024943 & 2.011 & 1.0034 \\
\hline SLC6A4 & Solute carrier family 6 (neurotransmitter transporter, serotonin), member 4 & NM_001045 & 2.001 & 4.5173 \\
\hline
\end{tabular}

The term 'cell surface receptor linked signal transduction' included several members of the $G$ protein-coupled receptor family (GPR128, GPR135, GPR132, GPR56 and GPR34). Indeed, GPR128 was the strongest upregulated gene in our microarray analysis (Table I). The $\mathrm{G}$ protein-coupled receptor (GPCR) superfamily has long been proposed to have vital dual roles in cellular adhesion and signaling (29). One of the best described GPCRs is GPR56. In addition to its role in neural progenitor cell migration (30), a role in suppression of tumor growth by the microenvironment have been investigated (31). By interacting with an extracellular matrix ligand, TG2 (transglutaminase 2), GPR56 seems to suppress tumor growth and metastasis in vivo; conversely, reduced expression is associated with tumor progression. In addition, it is overexpressed 
in many human glioblastomas and functions in tumor cell adhesion (32). SHC3, another component of this GO term, has been described in the literature. Miyake et al (18) observed a significantly higher level of ShcC protein in NBs with poor prognostic factors and indicated that the expression of ShcC potentially has a function in inhibiting the differentiation of NB cells (18).

Upregulation of 'cell adhesion' genes supports the hypothesis that NB-MSCs may undergo changes in their extracellular matrix and cell adhesion properties. Previous research has shown the modulation of NB cell differentiation by the extracellular matrix (33). In this study, the authors showed how extracellular matrix rigidity potentiates NB cell differentiation and decreases cell proliferation; and, as we mentioned above, the receptors of extracellular matrix molecules have been reported as markers of NB cell differentiation (17). Similarly, Chen et al (5) reported that suppression of cell adhesion proteins in NB tumors of stage $4^{+}$indicates the metastatic nature of this kind of NB tumors.

In addition, increased transcript levels of a number of relevant genes involved in cell-cycle regulation or apoptosis (e.g., CCND1, CDKN2A (p14/p16), RET and GDNF) suggest that there may be alterations in these processes in NB-MSCs. Similarly, the number of upregulated genes in the 'calcium ion binding' category may indicate that intracellular calcium is likely involved in the response of MSCs to NB. ANXA10 belongs to the annexin super-family of closely related calcium and membrane-binding proteins, and many studies have shown their potential role in tumor development and progression (34). An alternative explanation would be based in the evidence that NB cells stimulate osteoclasts to generate osteolytic lesions and set free calcium, in which interactions of NB cells with BM-derived MSCs play a critical role (35).

Finally, most of the downregulated GO terms contained genes encoding immune-related proteins. However, our results also showed upregulation of HLA-DRB5 and HLA-DOA1, which encode MHC class II molecules. In this sense, Johann et al (36) showed that NK cell cytotoxicity was significantly impaired after co-culturing NB cells with NB-MSCs, compared with MSCs of normal donors. Further study is needed to assess the impact of NB cancer cells on the immune response of MSCs.

In summary, we present initial data of a genome-wide analysis of MSCs from NB patients. Our data suggest that the microarray approach is a useful tool to identify deregulated genes in cultured MSCs isolated from NB patients. We provide preliminary genetic evidence of the interaction between MSCs and NB cancer cells in BM. Furthermore, we identifed relevant biological processes potentially altered in MSCs in response to NB. Future studies are necessary to connect these and other differentially expressed genes into their biological roles.

\section{Acknowledgements}

This study was supported by grants from the Fondo de Investigaciones Sanitarias (FIS; PI05/2217 and PI08/0029 to J.G.C.), MICINN (PLE2009-0115) and the Madrid Regional Government (S-BIO-0204-2006 and P2010/BMD-2420) in Spain. The experiments were approved by the appropriate committees.

\section{References}

1. Ehninger A and Trumpp A: The bone marrow stem cell niche grows up: mesenchymal stem cells and macrophages move in. J Exp Med 208: 421-428, 2011.

2. Mishra PJ, Glod JW and Banerjee D: Mesenchymal stem cells: flip side of the coin. Cancer Res 69: 1255-1258, 2009.

3. Hiyama E, Hiyama K, Yamaoka H, Sueda T, Reynolds CP and Yokoyama T: Expression profiling of favorable and unfavorable neuroblastomas. Pediatr Surg Int 20: 33-38, 2004.

4. Kamei N, Hiyama K, Yamaoka H, et al: Evaluation of genes identified by microarray analysis in favorable neuroblastoma. Pediatr Surg Int 25: 931-937, 2009.

5. Chen QR, Song YK, Yu LR, et al: Global genomic and proteomic analysis identifies biological pathways related to high-risk neuroblastoma. J Proteome Res 9: 373-382, 2010.

6. Abel F, Dalevi D, Nethander M, et al: A 6-gene signature identifies four molecular subgroups of neuroblastoma. Cancer Cell Int 11: 9, 2011.

7. Abarrategi A, Marinas-Pardo L, Mirones I, Rincon E and Garcia-Castro J: Mesenchymal niches of bone marrow in cancer. Clin Transl Oncol 13: 611-616, 2011.

8. Grisendi G, Bussolari R, Veronesi E, et al: Understanding tumorstroma interplays for targeted therapies by armed mesenchymal stromal progenitors: the Mesenkillers. Am J Cancer Res 1: 787-805, 2011.

9. Ma M, Ye JY, Deng R, Dee CM and Chan GC: Mesenchymal stromal cells may enhance metastasis of neuroblastoma via SDF-1/CXCR4 and SDF-1/CXCR7 signaling. Cancer Lett 312: $1-10,2011$.

10. Airoldi I, Cocco C, Morandi F, Prigione I and Pistoia V: CXCR5 may be involved in the attraction of human metastatic neuroblastoma cells to the bone marrow. Cancer Immunol Immunother 57: 541-548, 2008.

11. Dominici M, Le Blanc K, Mueller I, et al: Minimal criteria for defining multipotent mesenchymal stromal cells. The International Society for Cellular Therapy position statement. Cytotherapy 8: 315-317, 2006.

12. Tusher VG, Tibshirani R and Chu G: Significance analysis of microarrays applied to the ionizing radiation response. Proc Natl Acad Sci USA 98: 5116-5121, 2001.

13. Saeed AI, Sharov V, White J, et al: TM4: a free, open-source system for microarray data management and analysis. Biotechniques 34 : 374-378, 2003.

14. Huang dW, Sherman BT, Tan Q, et al: DAVID Bioinformatics Resources: expanded annotation database and novel algorithms to better extract biology from large gene lists. Nucleic Acids Res 35: W169-W175, 2007.

15. Hahn T, Or R, Bruchelt G, et al: Control of neuroblastoma cell proliferation and differentiation by human bone marrow. Cancer 77: 2614-2621, 1996.

16. Walton JD, Spengler BA, Biedler JL, Gerald WL, Cheung NKV and Ross RA: Microarray analyses of human neuroblastoma stem cells identifies genes associated with malignancy. Proc Amer Assoc Cancer Res 2005: 688, 2005.

17. Rozzo C, Chiesa V and Ponzoni M: Integrin upregulation as marker of neuroblastoma cell differentiation: correlation with neurite extension. Cell Death Differ 4: 713-724, 1997.

18. Miyake I, Ohira M, Nakagawara A and Sakai R: Distinct role of ShcC docking protein in the differentiation of neuroblastoma. Oncogene 28: 662-673, 2009.

19. Gery S, Sawyers CL, Agus DB, Said JW and Koeffler HP: TMEFF2 is an androgen-regulated gene exhibiting antiproliferative effects in prostate cancer cells. Oncogene 21: 4739-4746, 2002.

20. Lin K, Taylor JR, Wu TD, et al: TMEFF2 is a PDGF-AA binding protein with methylation-associated gene silencing in multiple cancer types including glioma. PLoS One 6: e18608, 2011.

21. Gesierich S, Paret C, Hildebrand D, et al: Colocalization of the tetraspanins, CO-029 and CD151, with integrins in human pancreatic adenocarcinoma: impact on cell motility. Clin Cancer Res 11: 2840-2852, 2005.

22. Zöller M: Tetraspanins: push and pull in suppressing and promoting metastasis. Nat Rev Cancer 9: 40-55, 2009.

23. Koshikawa K, Osada H, Kozaki K, et al: Significant upregulation of a novel gene, CLCP1, in a highly metastatic lung cancer subline as well as in lung cancers in vivo. Oncogene 21: 2822-2828, 2002.

24. Kim M, Lee KT, Jang HR, et al: Epigenetic downregulation and suppressive role of DCBLD2 in gastric cancer cell proliferation and invasion. Mol Cancer Res 6: 222-230, 2008. 
25. Rizvanov AA, Yalvaç ME, Shafigullina AK, et al: Interaction and self-organization of human mesenchymal stem cells and neuro-blastoma SH-SY5Y cells under co-culture conditions: A novel system for modeling cancer cell micro-environment. Eur J Pharm Biopharm 76: 253-259, 2010.

26. Maltman DJ, Hardy SA and Przyborski SA: Role of mesenchymal stem cells in neurogenesis and nervous system repair. Neurochem Int 59: 347-356, 2011.

27. Du W, Hozumi N, Sakamoto M, Hata J and Yamada T: Reconstitution of Schwannian stroma in neuroblastomas using human bone marrow stromal cells. Am J Pathol 173: 1153-1164, 2008.

28. Takashima Y,Era T, Nakao K, et al: Neuroepithelial cells supply an initial transient wave of MSC differentiation. Cell 129: 1377-1388, 2007.

29. Yona S, Lin HH, Siu WO, Gordon S and Stacey M: AdhesionGPCRs: emerging roles for novel receptors. Trends Biochem Sci 33: 491-500, 2008

30. Iguchi T, Sakata K, Yoshizaki K, Tago K, Mizuno N and Itoh H: Orphan $\mathrm{G}$ protein-coupled receptor GPR56 regulates neural progenitor cell migration via a $\mathrm{G}$ alpha $12 / 13$ and Rho pathway. J Biol Chem 283: 14469-14478, 2008.
31. Xu L and Hynes RO: GPR56 and TG2: possible roles in suppression of tumor growth by the microenvironment. Cell Cycle 6 : 160-165, 2007.

32. Shashidhar S, Lorente G, Nagavarapu U, et al: GPR56 is a GPCR that is overexpressed in gliomas and functions in tumor cell adhesion. Oncogene 24: 1673-1682, 2005.

33. Lam WA, Cao L, Umesh V, Keung AJ, Sen S and Kumar S Extracellular matrix rigidity modulates neuroblastoma cell differentiation and $\mathrm{N}$-myc expression. Mol Cancer 9: 35, 2010.

34. Mussunoor S and Murray GI: The role of annexins in tumor development and progression. J Pathol 216: 131-140, 2008.

35. Sohara Y, Shimada H, Minkin C, Erdreich-Epstein A, Nolta JA and DeClerck YA: Bone marrow mesenchymal stem cells provide an alternate pathway of osteoclast activation and bone destruction by cancer cells. Cancer Res 65: 1129-1135, 2005.

36. Johann PD, Vaegler M, Gieseke F, et al: Tumor stromal cells derived from paediatric malignancies display MSC-like properties and impair NK cell cytotoxicity. BMC Cancer 10: 501, 2010. 